PROCEEDINGS OF THE

AMERICAN MATHEMATICAL SOCIETY

Volume 140, Number 8, August 2012, Pages 2599-2612

S 0002-9939(2011)11107-9

Article electronically published on November 30, 2011

\title{
INVARIANT SUBSPACES OF THE LAWRENCE-KRAMMER REPRESENTATION
}

\author{
CLAIRE LEVAILLANT
}

(Communicated by Birge Huisgen-Zimmermann)

\begin{abstract}
The Lawrence-Krammer representation was used in 2000 to show the linearity of the braid group. The problem had remained open for many years. The fact that the Lawrence-Krammer representation of the braid group is reducible for some complex values of its two parameters is now known, as well as the complete description of these values. It is also known that when the representation is reducible, the action on a proper invariant subspace is an Iwahori-Hecke algebra action. In this paper, we prove a theorem of classification for the invariant subspaces of the Lawrence-Krammer space. We classify the invariant subspaces in terms of Specht modules. We fully describe them in terms of dimension and spanning vectors in the Lawrence-Krammer space.
\end{abstract}

\section{INTRODUCTION}

The Lawrence-Krammer representation $\mathcal{L K}_{n}$ of degree $\frac{n(n-1)}{2}$ was used in 2000 to show the linearity of the braid group on $n$ strands $(n \geq 3)$. This result is due to Bigelow in [1] and independently to Krammer in 6]. Their proofs are very different. Krammer's proof is algebraic while Bigelow's proof is topological. Linearity of a group means that there exists a faithful linear representation of this group. Thus, the group can be identified with a subgroup of $G L_{k}(F)$ for some field $F$ and some integer $k$. The Lawrence-Krammer representation first appears in a work of Lawrence in [7]. It is thus called the Lawrence-Krammer representation.

The Lawrence-Krammer representation, based on two parameters $t$ and $q$, was known by several authors (Cohen, Gijsbers, and Wales in [4, Marin in 12, Zinno in [18]) to be generically irreducible. It is shown in [10] with some restrictions on the parameter $q$ that when the two parameters are specialized to some nonzero complex numbers, the representation becomes reducible. The complete list of the nonzero complex parameters for which $\mathcal{L} \mathcal{K}_{n}$ is reducible is given in [10. In the same paper, it is shown that when $\mathcal{L K}_{n}$ is reducible, the action on a proper invariant subspace of the $\mathcal{L K}_{n}$ space is an Iwahori-Hecke algebra action. In this paper, we give the complete classification of the proper invariant subspaces of the $\mathcal{L} \mathcal{K}_{n}$ space in terms of Specht modules. Our result is as follows. It was originally stated as a conjecture in the author's Ph.D. thesis in 8 . The result also follows from Theorem 3.6

Received by the editors July 31, 2010 and, in revised form, January 28, 2011; February 15, 2011; and March 4, 2011.

2010 Mathematics Subject Classification. Primary 20F36; Secondary $20 \mathrm{C} 08$.

Key words and phrases. Braid groups, representation theory.

(C)2011 American Mathematical Society 
of [17, where Rui and Si use the notions of cellular algebras and Jucy-Murphy bases, which we do not use here.

Main Theorem. Let $n$ be an integer with $n \geq 3$. Assume that $q$ is not a $k$-th root of unity for every integer $k$ with $1 \leq k \leq n$. There are two cases:

(1) Assume that $q^{n} \neq-1$ when $t=-1$.

If $\mathcal{L K}_{n}$ is reducible, its unique proper invariant subspace is isomorphic to one of the Specht modules

$$
S^{(n)}, S^{(n-1,1)}, S^{(n-2,2)}, S^{(n-2,1,1)},
$$

which respectively arise if and only if

$$
t=\frac{1}{q^{n}}, t \in\left\{\frac{1}{\sqrt{q}^{n}},-\frac{1}{\sqrt{q}^{n}}\right\}, t=\frac{1}{q}, t=-1
$$

(2) If $t=-1$ and $q^{n}=-1$, there are exactly three proper invariant subspaces of the $\mathcal{L} \mathcal{K}_{n}$ space, and they are isomorphic to $S^{(n)}, S^{(n-2,1,1)}$ and $S^{(n)} \oplus S^{(n-2,1,1)}$ respectively.

In the next sections, we introduce the Lawrence-Krammer representation $\mathcal{L K}_{n}$ and prove the Main Theorem. Further, we fully describe all the proper invariant subspaces by providing their dimensions and some spanning vectors in the $\mathcal{L K}_{n}$ space.

\section{The Lawrence-Krammer Representation}

The Lawrence-Krammer space $\mathcal{V}^{(n)}$, abbreviated $\mathcal{L K}_{n}$ space, is the vector space of dimension $\frac{n(n-1)}{2}$ over the field $\mathbb{Q}(t, q)$ with spanning vectors the $x_{\beta}$ 's, indexed by the $\frac{n(n-1)}{2}$ positive roots $\beta$ 's of a root system of type $A_{n-1}$. In what follows, we will denote the simple roots by $\alpha_{1}, \ldots, \alpha_{n-1}$. The positive roots are the sums $\alpha_{i}+\cdots+\alpha_{j}$ (with $i \leq j$ ) of simple roots. If $\beta=\alpha_{i}+\cdots+\alpha_{j}$, we will denote the basis vector $x_{\beta}$ by $w_{i, j+1}$. The height $\operatorname{ht}(\beta)$ of a positive root $\beta$ is the sum of its coefficients with respect to the simple roots. These coefficients are either zeros or ones. The support $\operatorname{Supp}(\beta)$ of a positive root $\beta$ is the set of $k \in\{1, \ldots, n\}$ such that the coefficient of $\alpha_{k}$ in $\beta$ is nonzero. We will denote the set of positive roots by $\phi^{+}$. Finally, if $\left(m_{i j}\right)_{1 \leq i \leq j \leq n-1}$ denotes the Coxeter matrix of type $A_{n-1}$, the inner product between two simple roots $\alpha_{i}$ and $\alpha_{j}$ is given by

$$
\left(\alpha_{i} \mid \alpha_{j}\right)=-\cos \left(\frac{\pi}{m_{i j}}\right) .
$$

The Lawrence-Krammer representation can be constructed via BMW algebras. These algebras are named after Birman, Murakami, and Wenzl. They were introduced by Birman and Wenzl in 2] in order to study the linearity of the braid groups, and were introduced independently by Murakami in [16]. They feature in many areas, including statistical mechanics, knot theory, and quantum group theory. The BMW algebra $B\left(A_{n-1}\right)$, or simply $B$ of type $A_{n-1}$ with parameters $l$ and $m$ as defined in [4, is the algebra over the field $\mathbb{Q}(l, m)$ with $(n-1)$ generators $g_{1}, \ldots, g_{n-1}$ which satisfy the braid relations, and other elements $e_{1}, \ldots, e_{n-1}$ that are related to the $g_{i}$ 's by

$$
m e_{i}=l\left(g_{i}^{2}+m g_{i}-1\right)
$$


The other defining relations that relate the elements $e_{i}$ 's and $g_{i}$ 's of the algebra are the following:

$$
\begin{aligned}
g_{i} e_{i} & =l^{-1} e_{i} & & \text { for all } i, \\
e_{i} g_{j} e_{i} & =l e_{i} & & \text { when }|i-j|=1 .
\end{aligned}
$$

The BMW algebra $B$ modulo the two-sided ideal $I_{1}=B e_{1} B$ is the Hecke algebra with generators $g_{1}, \ldots, g_{n-1}$ and relations the braid relations and the relations

$$
g_{i}^{2}+m g_{i}=1 \quad \text { for all } i .
$$

When $m$ is a given nonzero complex number, we let $r$ and $-\frac{1}{r}$ be the two nonzero complex roots of the polynomial $X^{2}+m X-1=0$. So, the nonzero complex numbers $m$ and $r$ are related by $m=\frac{1}{r}-r$. Up to a rescaling of the generators, the algebra $B / I_{1}$ is the Iwahori-Hecke algebra $\mathcal{H}_{F, r^{2}}(n)$ of the symmetric group $\operatorname{Sym}(n)$ with parameter $r^{2}$ over the field $F=\mathbb{Q}(l, r)$, as defined in [13.

In [8, the author uses the isomorphism between the BMW algebra and the tangle algebra of Morton and Traczyk (see [15]) to construct a representation $\nu^{(n)}$ of degree $\frac{n(n-1)}{2}$ of the BMW algebra of type $A_{n-1}$ inside the $\mathcal{L K}_{n}$ space $\mathcal{V}^{(n)}$ over $F$. She shows that as a representation of the braid group on $n$ strands and up to some change of parameters and some rescaling of the generators, this representation is equivalent to $\mathcal{L} \mathcal{K}_{n}$. The change of parameters is given by $l t=r^{3}$ and $q=\frac{1}{r^{2}}$. The representation $\nu^{(n)}$ is defined on the generators of the algebra by

$$
\begin{aligned}
& \nu^{(n)}: B \longrightarrow \operatorname{End}_{F}\left(\mathcal{V}^{(n)}\right), \\
& g_{i} \longmapsto \nu_{i}
\end{aligned}
$$

where $\nu_{i}$ is the endomorphism defined on the basis vectors $x_{\beta}$ 's by

$$
\nu_{i}\left(x_{\beta}\right)= \begin{cases}r x_{\beta} & \text { if } \operatorname{Supp}(\beta) \cap\{i-1, i, i+1\}=\emptyset \\ & \text { or }\{i-1, i, i+1\} \subseteq \operatorname{Supp}(\beta), \\ \frac{1}{l} x_{\beta} & \text { if } \beta=\alpha_{i}, \\ x_{\beta+\alpha_{i}} & \text { if } \beta=\alpha_{s}+\cdots+\alpha_{i-1} \text { with } s \leq i-1, \\ x_{\beta+\alpha_{i}}+m r^{h t(\beta)-1} x_{\alpha_{i}}-m x_{\beta} & \text { if } \beta=\alpha_{i+1}+\cdots+\alpha_{k} \text { with } k \geq i+1, \\ x_{\beta-\alpha_{i}}+\frac{m}{l r^{h t(\beta)-2}} x_{\alpha_{i}}-m x_{\beta} & \text { if } \beta=\alpha_{s}+\cdots+\alpha_{i} \quad \text { with } s \leq i-1, \\ x_{\beta-\alpha_{i}} & \text { if } \beta=\alpha_{i}+\cdots+\alpha_{k} \quad \text { with } k \geq i+1 .\end{cases}
$$

We now show that the representation $\nu^{(n)}$ is equivalent to the LawrenceKrammer representation of the BMW algebra defined by Cohen, Gijsbers, and Wales. In [4], the authors define $I_{2}$ as the two-sided ideal of $B$ generated by all the products $e_{i} e_{j}$ with $|i-j|>1$. For each irreducible representation $\theta$ of the Hecke algebra of type $A_{n-3}$, they construct a representation of $B / I_{2}$ of degree $\left|\phi^{+}\right| \operatorname{deg}(\theta)$. They show that these are all the irreducible representations of $I_{1} / I_{2}$. The Lawrence-Krammer representation of the BMW algebra is obtained for one of the two inequivalent representations of degree one of the Hecke algebra of type $A_{n-3}$. We next show that $\nu^{(n)}$ is irreducible and factors through $I_{2}$. The proof of the following result can be found in [8]. The assumption on $r$ is equivalent to assuming that $\mathcal{H}_{F, r^{2}}(n)$ is semisimple (see Corollary 3.4, page 48, of [13]). 
Theorem 2.1. Assume $r^{2 k} \neq 1$ for every integer $k$ with $1 \leq k \leq n$. When $n \geq 4$, $\nu^{(n)}$ is irreducible except when

$$
l \in\left\{r,-r^{3}, \frac{1}{r^{2 n-3}},-\frac{1}{r^{n-3}}, \frac{1}{r^{n-3}}\right\}
$$

is reducible.

$\nu^{(3)}$ is irreducible except when

$$
l \in\left\{-r^{3}, \frac{1}{r^{3}},-1,1\right\}
$$

is reducible.

This result shows in particular that $\nu^{(n)}$ is generically irreducible for every integer $n$ with $n \geq 3$. Moreover, straightforward computations show that for every pair of nodes $i$ and $j$ with $|i-j|>1$, we have $\nu^{(n)}\left(e_{i} e_{j}\right)=0$. Thus, $\nu^{(n)}$ is an irreducible representation of $I_{1} / I_{2}$ of degree $\left|\phi^{+}\right|$. It must then be equivalent to the LawrenceKrammer representation of the BMW algebra of [4]. Our $r$ is the $\frac{1}{r}$ of 4 .

\section{The invariant subspaces of the $\mathcal{L K}_{n}$ SPaCe}

When the representation $\nu^{(n)}$ is reducible, the action on a proper invariant subspace of $\mathcal{V}^{(n)}$ is an Iwahori-Hecke algebra action. This is Proposition 1 of [1]. The following two theorems stated here for the Iwahori-Hecke algebra of the symmetric group $\operatorname{Sym}(n)$ instead of the symmetric group $\operatorname{Sym}(n)$ are due to James in [5]. In characteristic zero, when the Iwahori-Hecke algebra of the symmetric group is semisimple, they remain true for the Iwahori-Hecke algebra of the symmetric group (see [13]).

Theorem 3.1. Let $n$ be an integer with $n \geq 7$ and assume that $\mathcal{H}_{F, r^{2}}(n)$ is semisimple. Then, every irreducible $\mathcal{H}_{F, r^{2}}(n)$-module is either isomorphic to one of the Specht modules

$$
S^{(n)}, S^{\left(1^{n}\right)}, S^{(n-1,1)}, S^{\left(2,1^{n-2}\right)}
$$

or has dimension greater than $(n-1)$.

Theorem 3.2. Let $n$ be an integer with $n \geq 9$ and assume that $\mathcal{H}_{F, r^{2}}(n)$ is semisimple. Then, every irreducible $\mathcal{H}_{F, r^{2}}(n)$-module is either isomorphic to one of the Specht modules

$$
S^{(n)}, S^{(n-1,1)}, S^{(n-2,2)}, S^{(n-2,1,1)}
$$

or their conjugates, or has dimension greater than $\frac{(n-1)(n-2)}{2}$.

Theorem 3.1 fails for $n=4$ as $S^{(2,2)}$ has dimension 2 and for $n=6$ as $S^{(3,3)}$ and $S^{(2,2,2)}$ have dimension 5 .

Theorem 3.2 fails for $n=7$ as $S^{(4,3)}$ and $S^{(2,2,2,1)}$ have dimension 14 and for $n=8$ as $S^{(4,4)}$ and $S^{(2,2,2,2)}$ have dimension 14 .

A consequence of Theorem 3.1 and Theorem 3.2 is the following corollary.

Corollary 3.3. Let $n$ be an integer with $n \geq 5$ and $n \neq 8$ and assume that $\mathcal{H}_{F, r^{2}}(n)$ is semisimple. Then, the irreducible $\mathcal{H}_{F, r^{2}}(n)$-modules have dimension $1, n-1$, $\frac{n(n-3)}{2}, \frac{(n-1)(n-2)}{2}$, or dimension greater than $\frac{(n-1)(n-2)}{2}$.

Assume that $\mathcal{H}_{F, r^{2}}(8)$ is semisimple. Then, the irreducible $\mathcal{H}_{F, r^{2}}(8)$-modules have dimension 1, 7, 14, 20, 21 or dimension greater than 21 . 
We now recall some results of [11] about the existence of a one-dimensional invariant subspace of the $\mathcal{L K}_{n}$ space and of an irreducible $(n-1)$-dimensional invariant subspace of the $\mathcal{L} \mathcal{K}_{n}$ space for some values of the parameters $l$ and $r$.

Theorem 3.4. Let $n$ be an integer with $n \geq 3$ and assume $\mathcal{H}_{F, r^{2}}(n)$ is semisimple.

(i) Suppose $n \geq 4$. There exists a one-dimensional invariant subspace of $\mathcal{V}^{(n)}$ if and only if $l=\frac{1}{r^{2 n-3}}$. If so, it is spanned by

$$
\sum_{1 \leq s<t \leq n} r^{s+t} w_{s t}
$$

(ii) There exists a one-dimensional invariant subspace of $\mathcal{V}^{(3)}$ if and only if $l=\frac{1}{r^{3}}$ or $l=-r^{3}$. If $r^{6} \neq-1$, it is unique and

$$
\begin{aligned}
& \text { when } l=\frac{1}{r^{3}}, \quad \text { it is spanned by } w_{12}+r w_{13}+r^{2} w_{23}, \\
& \text { when } l=-r^{3}, \quad \text { it is spanned by } w_{12}-\frac{1}{r} w_{13}+\frac{1}{r^{2}} w_{23} .
\end{aligned}
$$

If $r^{6}=-1$, there are exactly two one-dimensional invariant subspaces of $\mathcal{V}^{(3)}$, and they are respectively spanned by the vectors above.

Proof. This is Theorem 4 of [11. It is in particular shown in the proof that, except when $n=3$, the Specht module $S^{(n)}$ occurs in the $\mathcal{L} \mathcal{K}_{n}$ space $\mathcal{V}^{(n)}$ for $l=\frac{1}{r^{2 n-3}}$, while the conjugate Specht module $S^{\left(1^{n}\right)}$ cannot occur in the $\mathcal{L} \mathcal{K}_{n}$ space. When $n=3, S^{(3)}$ occurs for the value $l=\frac{1}{r^{3}}$ and $S^{(3-2,1,1)}$ occurs for the value $l=-r^{3}$.

Theorem 3.5. Let $n$ be an integer with $n \geq 3$ and $n \neq 4$. Assume $\mathcal{H}_{F, r^{2}}(n)$ is semisimple. Then, there exists an irreducible $(n-1)$-dimensional invariant subspace of $\mathcal{V}^{(n)}$ if and only if $l=\frac{1}{r^{n-3}}$ or $l=-\frac{1}{r^{n-3}}$. If so, it is spanned by the $v_{i}^{(n)}$ 's, $1 \leq i \leq n-1$, where $v_{i}^{(n)}$ is defined by the formula

$$
\begin{aligned}
& v_{i}^{(n)}=\left(\frac{1}{r}-\frac{1}{l}\right) w_{i, i+1}+\sum_{s=i+2}^{n} r^{s-i-2}\left(w_{i, s}-\frac{1}{r} w_{i+1, s}\right) \\
&+ \epsilon_{l} \sum_{t=1}^{i-1} r^{n-i-2+t}\left(w_{t, i}-\frac{1}{r} w_{t, i+1}\right) \\
& \text { with }\left\{\begin{array}{l}
\epsilon_{\frac{1}{r^{n-3}}}=1, \\
\epsilon_{-\frac{1}{r^{n-3}}}=-1 .
\end{array}\right.
\end{aligned}
$$

Case $n=4$. Assume $\mathcal{H}_{F, r^{2}}(4)$ is semisimple. Then, there exists an irreducible 3 -dimensional invariant subspace of $\mathcal{V}^{(4)}$ if and only if $l \in\left\{\frac{1}{r},-\frac{1}{r},-r^{3}\right\}$.

If $l \in\left\{-\frac{1}{r}, \frac{1}{r}\right\}$, it is spanned by $v_{1}^{(4)}, v_{2}^{(4)}, v_{3}^{(4)}$.

If $l=-r^{3}$, it is spanned by the vectors

$$
\left\{\begin{array}{rrr}
u_{1}= & r w_{23}+w_{13}+\left(\frac{1}{r}+\frac{1}{r^{3}}\right) w_{34}-w_{24}-\frac{1}{r} w_{14}, \\
u_{2}= & -r w_{12}-r^{2} w_{13}-\frac{1}{r} w_{34}-\frac{1}{r^{2}} w_{24}+\left(r+\frac{1}{r}\right) w_{14}, \\
u_{3}= & \left(r+r^{3}\right) w_{12}+\frac{1}{r} w_{23}-w_{13}+w_{24}-r w_{14} .
\end{array}\right.
$$

Proof. This is Theorem 5 of [11. In particular, it is shown that for $n \geq 3$ and $n \neq 4$, $S^{(n-1,1)}$ occurs in the $\mathcal{L} \mathcal{K}_{n}$ space $\mathcal{V}^{(n)}$ for $l=\frac{1}{r^{n-3}}$ and for $l=-\frac{1}{r^{n-3}}$, while the 
conjugate Specht module $S^{\left(2,1^{n-2}\right)}$ cannot occur in the $\mathcal{L} \mathcal{K}_{n}$ space. When $n=4$, $S^{(3,1)}$ occurs in $\mathcal{V}^{(4)}$ when $l \in\left\{\frac{1}{r},-\frac{1}{r}\right\}$ and $S^{(4-2,1,1)}$ occurs in $\mathcal{V}^{(4)}$ for $l=-r^{3}$. In the same proof, it is also shown that the Specht modules $S^{(3,3)}$ and its conjugate $S^{(2,2,2)}$ both of dimension 5 cannot occur inside $\mathcal{V}^{(6)}$.

Remark 3.6. For $n \geq 5$, Theorem 3.4, Theorem 3.5 and Corollary 3.3 imply that if $\nu^{(n)}$ is reducible and if $l \notin\left\{\frac{1}{r^{n-3}},-\frac{1}{r^{n-3}}, \frac{1}{r^{2 n-3}}\right\}$, then an irreducible invariant subspace of $\mathcal{V}^{(n)}$ must have dimension greater than or equal to $\frac{n(n-3)}{2}$ when $n \neq 8$ and greater than or equal to 14 when $n=8$.

In fact, we have the following theorem.

Theorem 3.7. Let $n$ be an integer with $n \geq 4$ and assume that $\mathcal{H}_{F, r^{2}}(n)$ is semisimple. Then, there exists an irreducible $\frac{n(n-3)}{2}$-dimensional invariant subspace of $\mathcal{V}^{(n)}$ if and only if $l=r$. If so it is unique.

Definition 3.8. We introduce the $B$-module $K(n)=\bigcap_{1<i<j<n} \operatorname{Ker} \nu^{(n)}\left(C_{i j}\right)$, where $C_{i, i+1}=e_{i}$ and $C_{i j}=g_{j-1}^{-1} \cdots g_{i+1}^{-1} e_{i} g_{i+1} \cdots g_{j-1}$ for all $j \geq i+2$. We denote its dimension by $k(n)$.

Remark 3.9. Since the $e_{i}$ 's act trivially on any proper invariant subspace $\mathcal{W}$ of $\mathcal{V}^{(n)}$, such a space $\mathcal{W}$ must be contained in $K(n)$.

Proof. When $l=r$, we show that $K(n)$ is irreducible. For $n=4$, this result is part of Proposition 3 of [11. When $n \geq 5$, Proposition 4 of 11 shows that $K(n)$ is nonzero. Suppose first $n \neq 8$. If $K(n)$ is reducible, by semisimplicity of $\mathcal{H}_{F, r^{2}}(n)$, the $\mathcal{H}_{F, r^{2}}(n)$-module $K(n)$ decomposes as a direct sum $K_{1}(n) \oplus K_{2}(n)$ of $\mathcal{H}_{F, r^{2}}(n)$ modules with $K_{1}(n)$ irreducible. Since we have $\frac{n(n-1)}{2}-\frac{(n-1)(n-2)}{2}=n-1$ and $\frac{n(n-3)}{2}=\frac{(n-1)(n-2)}{2}-1$, Corollary 3.3 implies that one of the two modules $K_{1}(n)$ or $K_{2}(n)$ must have dimension less than or equal to $(n-1)$. We now recall that a necessary and sufficient condition on $r$ so that $\mathcal{H}_{F, r^{2}}(n)$ is semisimple is that $r^{2 k} \neq 1$ for every integer $k$ with $1 \leq k \leq n$. In particular, when $l=r$, we have $l \notin\left\{\frac{1}{r^{n-3}},-\frac{1}{r^{n-3}}, \frac{1}{r^{2 n-3}}\right\}$. Then, by Remark 3.6, it is impossible to have $K_{1}(n)$ or $K_{2}(n)$ of dimension less than or equal to $(n-1)$. Thus, $K(n)$ is irreducible and $k(n) \geq \frac{n(n-3)}{2}$ still by Remark 3.6. When $n=8$ the proof is the same but needs to be slightly adapted. We obtain that $K(8)$ is irreducible and $k(8) \geq 14$. A consequence of Remark 3.9 and of the irreducibility of $K(n)$ is that $K(n)$ is the unique proper invariant subspace of $\mathcal{V}^{(n)}$. For $n=4$, the result of Theorem 3.7 is Proposition 3 of [11. When $n \geq 5$ and $l=r$, it is shown in [10] that $k(n) \leq \frac{n(n-3)}{2}$ (see [10, proof of Theorem 3.3]; for a detailed proof, see pages 112-116 of [9]). Then, when $n \geq 5$ and $n \neq 8, k(n)=\frac{n(n-3)}{2}$. When $n=8$, we have $k(8) \in\{14,20\}$. But if $k(8)=14$, then we observe that $k(8)=k(7)$.

Lemma 3.10. Let $n$ be an integer with $n \geq 5$. Suppose $l=r$ and $\mathcal{H}_{F, r^{2}}(n-1)$ is semisimple. Then $K(n-1) \subseteq K(n)$.

Proof of the lemma. Let $n \geq 5$. The vector space $K(n) \cap \mathcal{V}^{(n-1)}$ is not the whole space $\mathcal{V}^{(n-1)}$ (for a proof, see the arguments of the proof of Proposition 1 of [11). Then by Remark 3.9, we have $K(n) \cap \mathcal{V}^{(n-1)} \subseteq K(n-1)$. Moreover, by Proposition 5 in Chapter 8 of $\left[8\right.$, we have $K(n) \cap \mathcal{V}^{(n-1)} \neq\{0\}$. Hence by irreducibility of $K(n-1)$, we must have $K(n) \cap \mathcal{V}^{(n-1)}=K(n-1)$. This implies in particular $K(n-1) \subseteq K(n)$. 
By the lemma, we get $K(8)=K(7)$. By Proposition 5 in Chapter 8 of [8, the element $r^{2} w_{12}-r w_{13}+w_{34}-r w_{24}$ belongs to $K(8)$. We act with $\nu_{7} \cdots \nu_{4}$ to see that the element $r^{4}\left(r^{2} w_{12}-r w_{13}\right)+w_{38}-r w_{28}$ also belongs to $K(8)$. However, this element is not in $K(7)$, so we get a contradiction. Hence it is impossible to have $k(8)=14$ and so $k(8)=20$. Thus, for all $n \geq 4$, we have shown that when $l=r$, the $B\left(A_{n-1}\right)$-module $K(n)$ is the unique proper invariant subspace of $\mathcal{V}^{(n)}$ and it has dimension $\frac{n(n-3)}{2}$.

Conversely, it is shown in 10] (see proof of Theorem 3.3) that if there exists an irreducible $\frac{n(n-3)}{2}$-dimensional invariant subspace of the $\mathcal{L} \mathcal{K}_{n}$ space, then $l=r$.

We now describe the irreducible $\frac{n(n-3)}{2}$-dimensional invariant subspace $K(n)$ of $\mathcal{V}^{(n)}$ when $l=r$.

Theorem 3.11. Assume $l=r$. Let $n$ be an integer with $n \geq 4$ and assume that $\mathcal{H}_{F, r^{2}}(n)$ is semisimple.

- When $n=4$, the unique invariant subspace $K(4)$ of $\mathcal{V}^{(4)}$ is spanned by the two linearly independent vectors

$$
\begin{aligned}
& w_{1}^{(4)}=\left(w_{14}-\frac{1}{r} w_{24}\right)+\left(w_{23}-r w_{13}\right), \\
& w_{2}^{(4)}=\left(w_{24}-\frac{1}{r} w_{34}\right)+\left(w_{13}-r w_{12}\right) .
\end{aligned}
$$

- When $n \geq 5$, the unique invariant subspace $K(n)$ of $\mathcal{V}^{(n)}$ is built inductively as a direct sum of the unique invariant subspace $K(n-1)$ of $\mathcal{V}^{(n-1)}$ and of an $(n-2)$-dimensional vector space spanned by the vectors

$$
\begin{aligned}
& w_{1}^{(n)}=w_{1, n}-\frac{1}{r} w_{2, n}+r^{n-4}\left(w_{23}-r w_{13}\right), \\
& w_{k}^{(n)}=w_{k, n}-\frac{1}{r} w_{k+1, n}+r^{n-4}\left(w_{1, k+1}-r w_{1, k}\right), \quad 2 \leq k \leq n-2 .
\end{aligned}
$$

Proof. When $n=4$, see Proposition 3 of [11. When $n \geq 5$, we have seen that $K(n-1) \subseteq K(n)$. Hence, it suffices to check that the $(n-2)$ linearly independent vectors of the theorem belong to $K(n)$. This is achieved in [8, Chapter 10].

We now study reducibility in the case $l=-r^{3}$. This case requires more attention. Indeed, when $r^{2 n}=-1$, we have $l=-r^{3}=\frac{1}{r^{2 n-3}}$. In that case, $K(n)$ is no longer irreducible. In fact we have the following result.

Theorem 3.12. Let $n$ be an integer with $n \geq 5$. Assume $\mathcal{H}_{F, r^{2}}(n)$ is semisimple.

(1) If $l=-r^{3}$ and $r^{2 n} \neq-1$, then $K(n)$ is irreducible and $k(n)=\frac{(n-1)(n-2)}{2}$. In particular, $K(n)$ is the unique proper invariant subspace of $\mathcal{V}^{(n)}$.

(2) If $l=-r^{3}$ and $r^{2 n}=-1$, then $K(n)$ is reducible and $k(n)=1+\frac{(n-1)(n-2)}{2}$. Moreover, $K(n)$ is a direct sum of the unique one-dimensional invariant subspace of $\mathcal{V}^{(n)}$ and of the unique irreducible $\frac{(n-1)(n-2)}{2}$-dimensional invariant subspace of $\mathcal{V}^{(n)}$.

Proof. When $r^{2 n} \neq-1$, the proof of irreducibility of $K(n)$ is the same as in the case $l=r$. Moreover, by [8, Lemma 10, Chapter 9], we know that $k(n) \leq \frac{(n-1)(n-2)}{2}$. If $n \neq 8$, we get $k(n)=\frac{(n-1)(n-2)}{2}$. When $n=8$, if $k(8)=14$, we have $7 \leq$ $\operatorname{dim}\left(K(8) \cap \mathcal{V}^{(7)}\right) \leq 14$. But since $-r^{3} \notin\left\{r,-\frac{1}{r^{4}}, \frac{1}{r^{4}}\right\}$, this is impossible. Hence the case $n=8$ is not an exception and $k(8)=21$.

When $r^{2 n}=-1$, we have $l=-r^{3}=\frac{1}{r^{2 n-3}}$. Then, there exists a one-dimensional invariant subspace of $\mathcal{V}^{(n)}$ by Theorem 3.4. We have $0 \subset K(n) \cap \mathcal{V}^{(n-1)} \subseteq K(n-1)$, 
where the first inclusion holds by [8, Chapter 8, Proposition 5]. Moreover, since $r^{2 n}=-1$, we have $r^{2(n-1)} \neq-1$. Thus by the first point, $K(n-1)$ is irreducible. It follows that $K(n) \cap \mathcal{V}^{(n-1)}=K(n-1)$. If $K(n)$ were one-dimensional, so would be $K(n) \cap \mathcal{V}^{(n-1)}$. Then $K(n-1)$ would also be one-dimensional. This would force $l=\frac{1}{r^{2 n-5}}$, which is impossible. Hence the one-dimensional invariant subspace of $\mathcal{V}^{(n)}$ has a summand $S$ in $K(n)$. In particular $K(n)$ is reducible. Moreover, except possibly when $n=8$, the uniqueness part in Theorem 3.4, and Theorems 3.5 and 3.7 allow us to claim that the summand $S$ has dimension greater than or equal to $\frac{(n-1)(n-2)}{2}$. As for $n=8$, if $\operatorname{dim}(S)=14$, then $k(8)=15$. By arguments already exposed before, we have $K(7) \subseteq K(8)$. Since by the first point we have $k(7)=15$, it follows that $K(7)=K(8)$. When $l=-r^{3}$, the vector

$$
-r w_{23}-\frac{1}{r} w_{34}+w_{24}
$$

belongs to $K(8)$ by [8, Chapter 8 , Proposition 5]. By acting with $\nu_{7} \nu_{6} \nu_{5} \nu_{4}$ on this vector, we see that

$$
-r^{5} w_{23}-\frac{1}{r} w_{38}+w_{28}
$$

also belongs to $K(8)$. The latter vector is not in $K(7)$, hence a contradiction. So in any case, we have $k(n) \geq 1+\frac{(n-1)(n-2)}{2}$. Further, from $K(n) \cap \mathcal{V}^{(n-1)}=K(n-1)$, we derive $k(n) \leq k(n-1)+(n-1)$. Replacing $k(n-1)=\frac{(n-2)(n-3)}{2}$, we get $k(n) \leq 1+\frac{(n-1)(n-2)}{2}$. Gathering both inequalities now yields $k(n)=1+\frac{(n-1)(n-2)}{2}$. From this equality on the dimensions, we deduce the existence of an irreducible $\frac{(n-1)(n-2)}{2}$-dimensional invariant subspace of $\mathcal{V}^{(n)}$. It remains to show that it is unique. Let $\mathcal{W}$ be an irreducible $\frac{(n-1)(n-2)}{2}$-dimensional invariant subspace of $\mathcal{V}^{(n)}$ such that $K(n)$ is a direct sum of $\mathcal{W}$ and of the one-dimensional invariant subspace of $\mathcal{V}^{(n)}$. Since for $n \geq 5$, we have $\frac{(n-1)(n-2)}{2}>n-1$, it follows that the intersection $\mathcal{W} \cap \mathcal{V}^{(n-1)}$ is nonzero. Then, by irreducibility of $K(n-1)$, we get $\mathcal{W} \cap \mathcal{V}^{(n-1)}=$ $K(n-1)$. In particular, $K(n-1) \subseteq \mathcal{W}$. Let $S$ be an $\mathcal{H}_{F, r^{2}}(n-1)$ summand of $K(n-1)$ in $\mathcal{W}$. Since $k(n-1)=\frac{(n-2)(n-3)}{2}$, this summand must be $(n-2)$ dimensional. To conclude, it will suffice to prove the following two lemmas.

Lemma 3.13. Let $n$ be an integer with $n \geq 5$. Assume that $\mathcal{H}_{F, r^{2}}(n)$ is semisimple. Suppose $l=-r^{3}$ and $r^{2 n}=-1$. In $K(n)$ there exists a unique one-dimensional $\mathcal{H}_{F, r^{2}}(n-1)$-module, namely the unique one-dimensional invariant subspace of $\mathcal{V}^{(n)}$.

Proof. This is an adaptation of Lemma 13, page 139 of [9], where the assumption $r^{2(n-1)}=-1$ on $r$ has been replaced with the assumption $r^{2 n}=-1$. The scalar $\mu$ of [9] must then take the value 1 instead of the value 0 . Thus, if such a space exists, it must be spanned by

$$
\sum_{1 \leq i<j \leq n} r^{i+j} w_{i j}
$$

This ends the proof of the lemma. A consequence of this lemma is that $S$ is an irreducible $\mathcal{H}_{F, r^{2}}(n-1)$-module of dimension $(n-2)$.

Lemma 3.14. Let $n$ be an integer with $n \geq 5$. Assume that $\mathcal{H}_{F, r^{2}}(n)$ is semisimple. Suppose $l=-r^{3}$ and $r^{2 n}=-1$. In $K(n)$ there exists a unique irreducible $\mathcal{H}_{F, r^{2}}(n-1)$-module of dimension $(n-2)$. 
Proof. The existence part is provided by the module $S$ above. The uniqueness part is more difficult and is treated in Proposition 17, page 145 of 9 .

This ends the proof of Theorem 3.12. We note that this theorem remains true for $n=4$.

The next theorem describes the irreducible $\frac{(n-1)(n-2)}{2}$-dimensional invariant subspace of $\mathcal{V}^{(n)}$ when $l=-r^{3}$.

Theorem 3.15. Let $n$ be an integer with $n \geq 4$ and assume $\mathcal{H}_{F, r^{2}}(n)$ is semisimple. Suppose $l=-r^{3}$.

When $n=4$, the irreducible 3-dimensional invariant subspace of $\mathcal{V}^{(4)}$ is spanned by the vectors $u_{1}, u_{2}, u_{3}$ of Theorem 3.5 .

When $n \geq 5$, the irreducible $\frac{(n-1)(n-2)}{2}$-dimensional invariant subspace of $\mathcal{V}^{(n)}$ is built inductively from the irreducible $\frac{(n-2)(n-3)}{2}$-dimensional invariant subspace of $\mathcal{V}^{(n-1)}$ by adding the $(n-2)$ linearly independent vectors:

$$
V_{k}^{(n)}=w_{k+1, n}-r w_{k, n}+r^{n-k} w_{k, k+1}, \quad k=1, \ldots, n-2 .
$$

Proof. First, we show a lemma.

Lemma 3.16. Let $n \geq 5$. The vectors $V_{1}^{(n)}, \ldots, V_{n-2}^{(n)}$ belong to $K(n)$. Moreover, the action of the $g_{i}$ 's on these vectors is as follows:

$$
\begin{aligned}
g_{k-1} \cdot V_{k}^{(n)} & =V_{k-1}^{(n)}+r V_{k}^{(n)}-r^{n-k-1} V_{k-1}^{(k+1)}, \\
g_{k} \cdot V_{k}^{(n)} & =-\frac{1}{r} V_{k}^{(n)}, \\
g_{k+1} \cdot V_{k}^{(n)} & =V_{k+1}^{(n)}+r V_{k}^{(n)}-r^{n-k-1} V_{k}^{(k+2)} \text { when } k<n-2, \\
g_{n-1} \cdot V_{n-2}^{(n)} & =-\frac{1}{r} V_{n-2}^{(n)}, \\
g_{n-1} \cdot V_{k}^{(n)} & =V_{k}^{(n-1)}-m V_{k}^{(n)} \quad \text { when } k<n-2, \\
g_{i} \cdot V_{k}^{(n)} & =r V_{k}^{(n)} \quad \text { when } i \notin\{k-1, k, k+1, n-1\} .
\end{aligned}
$$

Proof. The fact that these vectors belong to $K(n)$ is Claim 3, page 120 of [9]. The equalities that follow are obtained by straightforward computations.

We can now proceed to the proof of Theorem 3.15. Suppose $n \geq 5$. We distinguish between several cases.

(1) If $r^{2(n-1)} \neq-1$ and $r^{2 n} \neq-1$, then $K(n-1)$ is irreducible and has dimension $\frac{(n-2)(n-3)}{2}$. Also $K(n)$ is the irreducible $\frac{(n-1)(n-2)}{2}$-dimensional invariant subspace of $\mathcal{V}^{(n)}$. The irreducibility of $K(n-1)$ and the fact that $\frac{(n-1)(n-2)}{2}>n-1$ when $n \geq 5$ imply that $K(n-1) \subseteq K(n)$. Since we notice that $k(n)=k(n-1)+(n-2), K(n)$ is a direct sum of $K(n-1)$ and of an $(n-2)$-dimensional vector space spanned by the vectors $V_{1}^{(n)}, V_{2}^{(n)}$, $\ldots, V_{n-2}^{(n)}$.

(2) If $r^{2(n-1)}=-1$, then $r^{2 n} \neq-1$. So $K(n)$ is the irreducible $\frac{(n-1)(n-2)}{2}$ dimensional invariant subspace of $\mathcal{V}^{(n)}$. We have $0 \subset K(n) \cap \mathcal{V}^{(n-1)} \subseteq$ $K(n-1)$. Moreover, we know that $K(n-1)$ is a direct sum of a onedimensional invariant subspace and of an irreducible $\frac{(n-2)(n-3)}{2}$-dimensional 
invariant subspace of $\mathcal{V}^{(n-1)}$. Further, since by Lemma 13, page 139 of 9], there does not exist any one-dimensional $\mathcal{H}_{F, r^{2}}(n-1)$-module inside $K(n)$, we see that $K(n) \cap \mathcal{V}^{(n-1)}$ is irreducible, $\frac{(n-2)(n-3)}{2}$-dimensional. So, $K(n) \cap \mathcal{V}^{(n-1)}$ is the irreducible $\frac{(n-2)(n-3)}{2}$-dimensional invariant subspace of $\mathcal{V}^{(n-1)}$ and $K(n)$ is a direct sum of $K(n) \cap \mathcal{V}^{(n-1)}$ and of an $(n-2)$ dimensional vector space spanned by the vectors $V_{1}^{(n)}, V_{2}^{(n)}, \ldots, V_{n-2}^{(n)}$.

(3) If $r^{2 n}=-1$. Let $\mathcal{W}$ be the irreducible $\frac{(n-1)(n-2)}{2}$-dimensional invariant subspace of $\mathcal{V}^{(n)}$. We have seen along the proof of Theorem 3.12 that $\mathcal{W}$ contains $K(n-1)$, the irreducible $\frac{(n-2)(n-3)}{2}$-dimensional invariant subspace of $\mathcal{V}^{(n-1)}$. Consider the $F$-vector space

$$
\mathcal{S}=K(n-1) \oplus \operatorname{Span}_{F}\left(V_{1}^{(n)}, \ldots, V_{n-2}^{(n)}\right) .
$$

This vector space has dimension $\frac{(n-1)(n-2)}{2}$ over $F$. We will show that it is stable under the action by the $g_{i}$ 's and that $\mathcal{W}=\mathcal{S}$. When $r^{2 n}=-1$, we have $r^{2} \neq-1,\left(r^{2}\right)^{2} \neq-1, \ldots, r^{2(n-1)} \neq-1$. We notice that $K(3)$ is spanned over $F$ by $V_{1}^{(3)}$ (see Theorem 3.4) and a use of the computer program of Appendix $A$ of $\left[8\right.$ shows that the vectors $V_{1}^{(3)}, V_{1}^{(4)}$ and $V_{2}^{(4)}$ belong to $K(4)$. In particular, we have $K(3) \subset K(4)$. These remarks and point (1) of the ongoing proof imply that

$$
\left\{\begin{array}{l}
K(3) \subset K(4) \subset \cdots \subset K(n-1), \\
K(s)=\left\langle V_{i}^{(j)}: 3 \leq j \leq s, 1 \leq i \leq j-2\right\rangle_{F} .
\end{array}\right.
$$

Further, we have

\section{It follows that}

$$
\begin{array}{rll}
g_{n-1} \cdot V_{k}^{(j)} & =r V_{k}^{(j)} & \forall 3 \leq j \leq n-2, \forall 1 \leq k \leq j-2, \\
g_{n-1} \cdot V_{k}^{(n-1)} & =V_{k}^{(n)} & \forall 1 \leq k \leq n-3 .
\end{array}
$$

$$
g_{n-1} . K(n-1) \subseteq \mathcal{S} .
$$

This inclusion, point $(\star)$, and the equalities of Lemma 3.16 imply that $\mathcal{S}$ is stable under the action by the $g_{i}$ 's. Since $\mathcal{S}$ is contained in $K(n)$, it follows that $\mathcal{S}$ is an $\mathcal{H}_{F, r^{2}}(n)$-module. Moreover, by choice of $l$ and $r$, we see that $\mathcal{S}$ must be irreducible. Hence $\mathcal{S}$ is the irreducible $\frac{(n-1)(n-2)}{2}$-dimensional invariant subspace of $\mathcal{V}^{(n)}$. This ends the proof of Theorem 3.15.

\section{Proof of the Main Theorem}

We will work on the representation $\nu^{(n)}$ of this paper instead of the original representation of Krammer of [6]. This is allowed by the following lemma.

Lemma 4.1. It suffices to check the main theorem on the representation $\nu^{(n)}$ where $q$ has been replaced by $\frac{1}{r^{2}}$ and $t$ has been replaced by $\frac{r^{3}}{l}$.

Proof. We recall from $\S 2$ that the representation of this paper is equivalent to the Lawrence-Krammer representation of the BMW algebra of [4, where our $r$ is the $\frac{1}{r}$ of [4]. Further, up to some rescaling of the generators, the representation of [4] is equivalent, as a representation of the braid group on $n$ strands, to the representation of the Artin group of type $A_{n-1}$ of [3]. The parameters $t$ and $r$ of $[3]$ are related to 
the parameters $l$ and $r$ of $\left[4\right.$ by $l=\frac{1}{t r^{3}}$. The representation of [3] is itself equivalent to the original representation of Krammer of [6] with parameters $t$ and $q$. The link between the parameter $q$ of [6] and the parameter $r$ of [3] is given by $q=r^{2}$.

Proof of the main theorem. We first deal with the uniqueness part when we exclude the case when $l=-r^{3}$ and $r^{2 n}=-1$. Then, under the assumption that $\mathcal{H}_{F, r^{2}}(n)$ is semisimple, the values $\frac{1}{r^{2 n-3}}, \frac{1}{r^{n-3}},-\frac{1}{r^{n-3}}, r,-r^{3}$ are all distinct. When $l=r$ or $l=-r^{3}$, we have seen that $K(n)$ is irreducible. As any proper invariant subspace of $\mathcal{V}^{(n)}$ must be contained in $K(n)$ (this is Remark 3.9), the $B\left(A_{n-1}\right)$-module $K(n)$ is then the unique proper invariant subspace of $\mathcal{V}^{(n)}$. Next, suppose that $l \in\left\{\frac{1}{r^{n-3}},-\frac{1}{r^{n-3}}, \frac{1}{r^{2 n-3}}\right\}$ and let $\mathcal{W}$ be an irreducible invariant subspace of $\mathcal{V}^{(n)}$. If $\operatorname{dim}(\mathcal{W}) \geq \frac{(n-1)(n-2)}{2}$, then $\operatorname{dim}(\mathcal{W})>n-1$ as soon as $n \geq 5$. This implies that $\mathcal{W} \cap \mathcal{V}^{(n-1)} \neq\{0\}$, hence $\nu^{(n-1)}$ is reducible. Also, we have $\operatorname{dim}(\mathcal{W})>2 n-3$ as soon as $n \geq 6$. This implies that $\mathcal{W} \cap \mathcal{V}^{(n-2)} \neq\{0\}$, hence $\nu^{(n-2)}$ is reducible. Using Theorem 2.1 and our usual restriction on the parameter $r$, we then get $l \in\left\{r,-r^{3}\right\}$, a contradiction. By the same proof, when $n=8$, we cannot have $\operatorname{dim}(\mathcal{W})=14$ since $14>13$. So assuming $n \geq 6$ and using Corollary 3.3 and Theorem 3.7, we must hence have $\operatorname{dim}(\mathcal{W}) \in\{1, n-1\}$. Now the uniqueness follows from the uniqueness in Theorems 3.4 and 3.5. When $n=5$, the irreducible representations of $\mathcal{H}_{F, r^{2}}(5)$ have degrees $1,4,5$ and 6 . If $\operatorname{dim}(\mathcal{W})=6$, applying the reducibility theorem on $\nu^{(4)}$, we get $l \in\left\{r,-r^{3}, \frac{1}{r},-\frac{1}{r}, \frac{1}{r^{5}}\right\}$. Since we assumed $l \in\left\{\frac{1}{r^{7}}, \frac{1}{r^{2}},-\frac{1}{r^{2}}\right\}$, we then have $l=\frac{1}{r^{7}}=-\frac{1}{r}$. This means that there exists a (unique) one-dimensional invariant subspace in $\mathcal{V}^{(5)}$ and an irreducible 3-dimensional invariant subspace in $\mathcal{V}^{(4)}$, which is also the unique proper invariant subspace of $\mathcal{V}^{(4)}$. We get $k(5)=7$ and so $\operatorname{dim}\left(K(5) \cap \mathcal{V}^{(4)}\right) \geq 3$. But $\mathcal{V}^{(4)}$ has a unique proper invariant subspace which has dimension 3 , and so $K(5) \cap \mathcal{V}^{(4)}=K(4)$. In particular, we derive $K(4) \subseteq K(5)$. Further, by Theorem 3.5,

$$
v_{2}^{(4)}=\left(\frac{1}{r}+r\right) w_{23}+\left(w_{24}-\frac{1}{r} w_{34}\right)-r\left(w_{12}-\frac{1}{r} w_{13}\right)
$$

belongs to $K(4)$. However, when $l=-\frac{1}{r}$, the action of $C_{35}$ on $v_{2}^{(4)}$ is nonzero (use the tables of Appendix $C$ in [8]). We conclude that it is impossible to have $\operatorname{dim}(\mathcal{W})=6$. Thus, an invariant subspace is unique in the case when $n=5$ as well. This ends the uniqueness part in point number (1).

We now study the case when $l=-r^{3}$ and $r^{2 n}=-1$. As $K(n)$ is a direct sum of the unique one-dimensional invariant subspace and of the unique irreducible $\frac{(n-1)(n-2)}{2}$-dimensional invariant subspace of $\mathcal{V}^{(n)}$, there are indeed exactly three proper invariant subspaces in $\mathcal{V}^{(n)}$. It remains to show that when $n \geq 5$, the unique irreducible $\frac{(n-1)(n-2)}{2}$-dimensional invariant subspace of $\mathcal{V}^{(n)}$ is isomorphic to the Specht module $S^{(n-2,1,1)}$. This is the object of Proposition 18, page 149 of $[9$. We recall that as part of the proof of Theorem 3.4, the Specht module $S^{(1,1,1)}$ occurs in $\mathcal{V}^{(3)}$ when $l=-r^{3}$ and as part of the proof of Theorem 3.5, the Specht module $S^{(2,1,1)}$ occurs in $\mathcal{V}^{(4)}$ when $l=-r^{3}$. Point number (2) is thus entirely proven.

We continue further with point number (1). The unique proper invariant subspace of $\mathcal{V}^{(n)}$ is $K(n)$. We simply call it $\mathcal{W}$. When $l=\frac{1}{r^{2 n-3}}$, we know that $\mathcal{W}$ is isomorphic to $S^{(n)}$ by the proof of Theorem 3.4. Suppose next $l \in\left\{\frac{1}{r^{n-3}},-\frac{1}{r^{n-3}}\right\}$. Then $\mathcal{W}$ is isomorphic to $S^{(n-1,1)}$ by the proof of Theorem 3.5. Suppose now $l=r$. 
By Theorem 3.7, we know that $\mathcal{W}$ is an irreducible $\frac{n(n-3)}{2}$-dimensional $\mathcal{H}_{F, r^{2}}(n)$ module. By Theorem 3.2, such an irreducible $\mathcal{H}_{F, r^{2}}(n)$-module must be isomorphic to $S^{(n-2,2)}$ or its conjugate Specht module $S^{\left(2,2,1^{n-4}\right)}$, except when $n=7$ in which case it can also be isomorphic to $S^{(4,3)}$ or its conjugate Specht module $S^{(2,2,2,1)}$, both of dimension 14. Thus, there are two things to show. One of them is that for $n \geq 5$, the $\mathcal{H}_{F, r^{2}}(n)$-module $\mathcal{W}$ cannot be isomorphic to $S^{\left(2,2,1^{n-4}\right)}$. The other one is that $\mathcal{W}$ cannot be isomorphic to $S^{(4,3)}$ or to $S^{(2,2,2,1)}$ when $n=7$. We start with the first point and proceed by induction on $n \geq 5$ to show that if $\mathcal{W}$ is an irreducible $\frac{n(n-3)}{2}$-dimensional invariant subspace of $\mathcal{V}^{(n)}$, it is impossible to have $\mathcal{W} \simeq S^{\left(2,2,1^{n-4}\right)}$. We show that this is true when $n=5$. This is Result 1 on page 21 of [11. When $n \geq 6$, we use the branching rule. If $\mathcal{W} \simeq S^{\left(2,2,1^{n-4}\right)}$, then

$$
\mathcal{W} \downarrow_{\mathcal{H}_{F, r^{2}}(n-1)} \simeq S^{\left(2,1^{n-3}\right)} \oplus S^{\left(2,2,1^{n-5}\right)} .
$$

We have $0 \subset \mathcal{W} \cap \mathcal{V}^{(n-1)} \subset \mathcal{W} \downarrow_{\mathcal{H}_{F, r^{2}}(n-1)}$, so we get $\mathcal{W} \cap \mathcal{V}^{(n-1)} \simeq S^{\left(2,2,1^{n-5}\right)}$. This is in contradiction with our induction hypothesis, and we are done with the first point. We now deal with the second point. First, if $\mathcal{W}$ is isomorphic to $S^{(2,2,2,1)}$, then by the branching rule,

$$
\mathcal{W} \downarrow_{\mathcal{H}_{F, r^{2}}(6)} \simeq S^{(2,2,2)} \oplus S^{(2,2,1,1)} .
$$

Since by the proof of Theorem 3.5, the Specht module $S^{(2,2,2)}$ cannot occur in $\mathcal{V}^{(6)}$, we must have $\mathcal{W} \cap \mathcal{V}^{(6)} \simeq S^{(2,2,1,1)}$. Another application of the branching rule then yields

$$
\mathcal{W} \cap \mathcal{V}^{(6)} \downarrow_{\mathcal{H}_{F, r^{2}}(5)} \simeq S^{(2,1,1,1)} \oplus S^{(2,2,1)} .
$$

The intersection $\mathcal{W} \cap \mathcal{V}^{(5)}$ is nonzero. Moreover, $S^{(2,1,1,1)}$ does not occur in $\mathcal{V}^{(5)}$ by the proof of Theorem 3.5. Also $S^{(2,2,1)}$ does not occur in $\mathcal{V}^{(5)}$ (this is Result 1 on page 21 of [11]). We thus get a contradiction. If now $\mathcal{W}$ is isomorphic to $S^{(4,3)}$, applying the branching rule twice yields

$$
\mathcal{W} \downarrow_{\mathcal{H}_{F, r^{2}}(5)} \simeq 2 S^{(3,2)} \oplus S^{(4,1)} .
$$

Let $w_{1}, w_{2}, w_{3}, w_{4}, w_{5}$ be linearly independent vectors of $\mathcal{W}$ such that the actions by $g_{1}, g_{2}, g_{3}$, and $g_{4}$ on these vectors is given by the matrices $P_{i}$ 's of Fact 1 on page 19 of [11. Notice the presence of a seventh node does not change the computations of [11. Indeed, since $g_{1} \cdot w_{4}=-\frac{1}{r} w_{4}$ and $g_{3} \cdot w_{4}=-\frac{1}{r} w_{4}$, we see that the vector $w_{4}$ belongs to $\mathcal{V}^{(4)}$. Then the relations $w_{5}=g_{4} . w_{4}, w_{1}=g_{2} . w_{4}-r w_{4}, w_{2}=g_{4} \cdot w_{1}$, $w_{3}=g_{3} \cdot w_{2}-r w_{2}$, show that the presence of a seventh node can just be forgotten. Then, up to a multiplication by a scalar, the $w_{i}$ 's are uniquely determined as in [11. This contradicts the multiplicity of $S^{(3,2)}$ in $(\diamond)$. So we are done with the case $l=r$. It remains to deal with the reducibility case $l=-r^{3}$ when we assume $r^{2 n} \neq-1$. In this case $\mathcal{W}$ is an irreducible $\frac{(n-1)(n-2)}{2}$-dimensional $\mathcal{H}_{F, r^{2}}(n)$-module. A use of Theorem 3.2 shows that $\mathcal{W}$ must be isomorphic to $S^{(n-2,1,1)}$ or to $S^{\left(3,1^{n-3}\right)}$. We show that $\mathcal{W}$ is isomorphic to $S^{(n-2,1,1)}$. This is already true for $n=3$ by the proof of Theorem 3.4 and for $n=4$ by the proof of Theorem 3.5. When $n=5$, there is nothing to prove as $S^{(3,1,1)}$ is self-conjugate. As for $n \geq 6$, we proceed by 
induction on $n$ to show that $S^{\left(3,1^{n-3}\right)}$ cannot occur in $\mathcal{V}^{(n)}$. First we show that $S^{(3,1,1,1)}$ cannot occur in $\mathcal{V}^{(6)}$. Suppose $K(6) \simeq S^{(3,1,1,1)}$. Then by the branching rule,

$$
K(6) \downarrow_{\mathcal{H}_{F, r^{2}}(4)} \simeq 2 S^{(2,1,1)} \oplus S^{(3,1)} \oplus S^{(1,1,1,1)} .
$$

In particular, there exists a vector $w$ of $K(6)$ such that

$$
g_{i} \cdot w=-\frac{1}{r} w \text { for each } i \in\{1,2,3\} .
$$

These relations imply that in $w$ there are no terms in $w_{1 j}$ or $w_{2 j}$ or $w_{3 j}$ or $w_{4 j}$ or $w_{j k}$ for any $j \geq 5$ and any $k \geq j+1$. In other words, $w$ belongs to $\mathcal{V}^{(4)}$. But in $\mathcal{V}^{(4)}$ there does not exist any one-dimensional invariant subspace isomorphic to $S^{\left(1^{4}\right)}$, hence a contradiction. This finishes the base case $n=6$. Let $n \geq 7$ and suppose that $S^{\left(3,1^{n-4}\right)}$ cannot occur inside $\mathcal{V}^{(n-1)}$. We show that $S^{\left(3,1^{n-3}\right)}$ cannot occur inside $\mathcal{V}^{(n)}$. If $\mathcal{W}$ is an irreducible $\frac{(n-1)(n-2)}{2}$-dimensional invariant subspace of $\mathcal{V}^{(n)}$ that is isomorphic to $S^{\left(3,1^{n-3}\right)}$, its restriction to $\mathcal{H}_{F, r^{2}}(n-1)$ is isomorphic to $S^{\left(3,1^{n-4}\right)} \oplus S^{\left(2,1^{n-3}\right)}$. Then $\mathcal{W} \cap \mathcal{V}^{(n-1)}$ is isomorphic to $S^{\left(3,1^{n-4}\right)}$. This is impossible with our induction hypothesis. This ends the proof of the Main Theorem.

\section{REFERENCES}

1. S.J. Bigelow, Braid groups are linear, J. Amer. Math. Soc. 14 (2001), 471-486. MR 1815219 (2002a:20043)

2. J.S. Birman and H. Wenzl, Braids, link polynomials and a new algebra, Trans. Amer. Math. Soc. 313 (1989), no. 1, 249-273. MR992598(90g:57004)

3. A.M. Cohen and D.B. Wales, Linearity of Artin groups of finite type, Isr. J. Math. 131 (2002), 101-123. MR.1942303 (2003j:20062)

4. A.M. Cohen, D.A.H. Gijsbers and D.B. Wales, BMW algebras of simply laced type, J. Algebra 286 (2005), no. 1, 107-153. MR2124811 (2006f:16047)

5. G.D. James, On the minimal dimensions of irreducible representations of symmetric groups, Math. Proc. Camb. Phil. Soc. 94 (1983), 417-424. MR720791 (86c:20018)

6. D. Krammer, Braid groups are linear, Ann. of Math. (2) 155 (2002), 131-156. MR.1888796 (2003c:20040)

7. R. Lawrence, Homological representations of the Hecke algebra, Comm. Math. Physics 135 (1990), 141-191. MR.1086755 (92d:16020)

8. C. Levaillant, Irreducibility of the Lawrence-Krammer representation of the BMW algebra of type $A_{n-1}$, Ph.D. thesis, California Institute of Technology (2008), http://thesis.library. caltech.edu/2255/1/thesis.pdf

9. C. Levaillant, Irreducibility of the Lawrence-Krammer representation of the BMW algebra of type $A_{n-1}$, arXiv:0901.3908 (arXiv version of the Ph.D. thesis).

10. C. Levaillant, Irreducibility of the Lawrence-Krammer representation of the BMW algebra of type $A_{n-1}$, C.R. Acad. Sci. Paris, Ser. I 347 (2009), 15-20. MR.2536742 (2010h:20078)

11. C.I. Levaillant and D.B. Wales, Parameters for which the Lawrence-Krammer representation is reducible, arXiv:0901.3856. (Shorter version available in J. Algebra 323 (2010), 1966-1982. MR.2594657 (2011d:20075))

12. I. Marin, Sur les représentations de Krammer génériques, Ann. Inst. Fourier (Grenoble) $\mathbf{5 7}$ (2007), no. 6, 1883-1925. MR2377890(2009d:20083)

13. A. Mathas, Iwahori-Hecke algebras and Schur algebras of the symmetric group, University Lecture Series, Vol. 15, Amer. Math. Soc., Providence, RI, 1999. MR.1711316 (2001g:20006)

14. J.A. Moody, The Burau representation of the braid group $B_{n}$ is unfaithful for large $n$, Bull. Amer. Math. Soc. (N.S.) 25 (1991), no. 2, 379-384. MR1098347 (92b:20041)

15. H.R. Morton and A.J. Wasserman, A basis for the Birman-Wenzl algebra, preprint, 1989. 
16. J. Murakami, The Kauffman polynomial of links and representation theory, Osaka J. Math. 24 (1987), 745-758. MR927059 (89c:57007)

17. H. Rui and M. Si, Blocks of Birman-Murakami-Wenzl algebras, Int. Math. Res. Notices IMRN 2011, no. 2, 452-486. MR2764870

18. M. Zinno, On Krammer's representation of the braid group, Math. Ann. 321 (2001), 197-211. MR:1857374 (2002h:20055)

Department of Mathematics, Caltech, Pasadena, California 91125

E-mail address: cl@caltech.edu

Current address: Department of Mathematics, University of California Santa Barbara, Santa Barbara, California 93106

E-mail address: claire@math.ucsb.edu 